\title{
A New Methodology for Crowd Estimation: Linear Quadratic Estimation
}

\author{
Jugal Kishor Gupta \\ Research Scholar, \\ IFTM University \\ Moradabad, India
}

\author{
S.K. Gupta, PhD \\ Assistant Professor \\ Department of Computer Science \& Engineering \\ Bundelkhand Institute of Engineering \& \\ Technology \\ Jhansi, India
}

\begin{abstract}
Recently, crowd estimation techniques in real-time are more popular research field using computer vision. here understand the behavior of the system using Linear Quadratic Estimation or kalman filter with new proposed index parameter which will help to understand the accuracy of the system still no more parameter discover to judge the accuracy of the system which is us to estimate the crowd or tracking the crowd. Crowd estimation does play an very critical role in intelligent crowd monitoring. All results have been implemented in MATLAB R2013.
\end{abstract}

\section{Keywords}

crowd, filter, parameter, kalman and Linear Quadratic Estimation.

\section{INTRODUCTION}

How can we control crowd? The answer of this question is crtical because without knowing the estimation how can we plan to manage the crowd for human safety. Since when an accident happens where there is congestion of people many lives can be lost. Freshly routine detection of people and accepting their behaviors from images became a very significant research field.

Two significant aspects of the problem of correct management and control of crowds are the design of environments where crowd congestion is expected to arise and the real-time monitoring of crowds within existing, typically urban, structures. The development of models of crowd behaviour provides a basis for informing architects and town planners to design safer buildings. Sime [2] reviews crowd psychology in terms of its relationship to engineering and crowd safety and stresses the need to validate computer simulations of crowd movement and escape behaviour against psychological as well as engineering criteria.

\section{PREVIOUS TECHNIQUE FOR AUTOMATIC ESTIMATION OF CROWD DENSITY}

Ming Jiang et.al. proposed a methodology for crowd density estimation, which is mixture of the pixel statistical feature and texture feature. The proposed methodology removed background with Gaussian mixture model and gave a first round judgment for the crowd density through pixel feature, meanwhile reduced the impact of outlook distortion by dividing the region of interest. The texture features were extracted using GLCM, and selected Contrast $0^{\circ}$ and Homogeneity $0^{\circ}$ as texture feature.

Results are effective for experimental and comparative technique both, universal method which can be used in a real- time crowd density estimation system. And this paper estimated the crowd size for high density and extremely high density, which was more conducive to group events analysis.

Certainly, there is still much room to improve the accuracy. If the image contains crowd shadow and reflective surface, it might lead to misclassification. In the future, we will accelerate foreground detection approach and try to eliminate shadow noise in the image.[2]

Marana et.al. proposed two technique which is based on different approaches of texture analysis, first technique is statistical and another technique is spectral, are applied on real images captured in an area of Liverpool Street Railway Station, London, UK. The results obtained show that both methods present similar general rates of correct estimation, and that the potential use of texture description for the problem of automatic estimation of crowd densities is encouraging.[3]

Dridi proposed three methods manual, semi-automatic and automatic for tracking individual targets in high density crowd scenes where thousands of people are gathered. The necessary data about the motion of individuals and a lot of other physical information can be extracted from consecutive image sequences in different ways, including optical flow and block motion estimation. One of the famous methods for tracking moving objects is the block matching method.

This method of subject motion which is requiring the condition of a comparison window through which we can determines the scale of the estimate. In this paper, we present a real-time method for tracking system or sequence of highly resolution image or viedo obtained by image acquisition device like camera or CCTV which is placed in different crowded places like cinema, railway station and in tradition mela. The objective is to estimate ordinary velocities as a function of the local density. The resulting data of tracking moving foottravelers based on video sequences are presented in the following section. Through the evaluated system the spatiotemporal coordinates of each pedestrian during the Tawaf ritual are established. The pilgrim velocities as function of the local densities in the Mataf area (Haram Mosque Mecca) are illustrated and very precisely documented. Tracking where density are 7-8 person per meter square is extremely critical or challenging due to the small number of pixels on the target, appearance doubt resulting from the dense packing, and severe inter-object occlusions. The tracking method comparison which is illustrated in this paper overcomes these challenges by using a virtual camera which is matched in position, rotation and focal length to the original camera in such a way that the features of the 3D-model match the feature position of the filmed mosque. In this model an individual feature has to be identified by eye, where contrast is a criterion. 
We do know that the travelers walk on a plane, and after matching the camera we also have the height of the plane in 3D-space from our 3D-model. A point object is placed at the position of a selected ordinary. During the animation we set multiple animation-keys (approximately every 25 to 50 frames which equals 1 to 2 seconds) for the position, such that the position of the point and the pedestrian overlay nearly at every time. By combining all these variables with the available appearance information, we are able to track individual targets in high density crowds [4].

\section{KALMAN FILTER}

The Kalman filter can use in image processing with multiple applications with MATLAB. In various applications of kalman filter control of vechical, spacecraft, guidance of navigation , and particularly aircraft are most frequently use in image procesing. Furthermore, in digital signal processing the Kalman filter is a used for analysis the time or frequency domain, in our paper Kalman filters use to track the object which is one of the feature of Kalman filter, kalman filter can also work in the field of robotic motion planning and control, and they are sometimes included in trajectory optimization. The multi-fractional order estimator is a simple and practical alternative to the Kalman filter for tracking targets. In Kalman filter cycle there are two block which is use to give the input to the system i.e System model and sensor. System Model gives two input observation model and motion model to measurement predication and state prediction respectively. State prediction predicted the state and send to the measurement prediction, after prediction the measurement give the message to data association, data association update the data of the basic of target detection which sense the data from input sensors and data association process is iterative until the desired output is not achieved. In our paper only result of motion models are discussed.

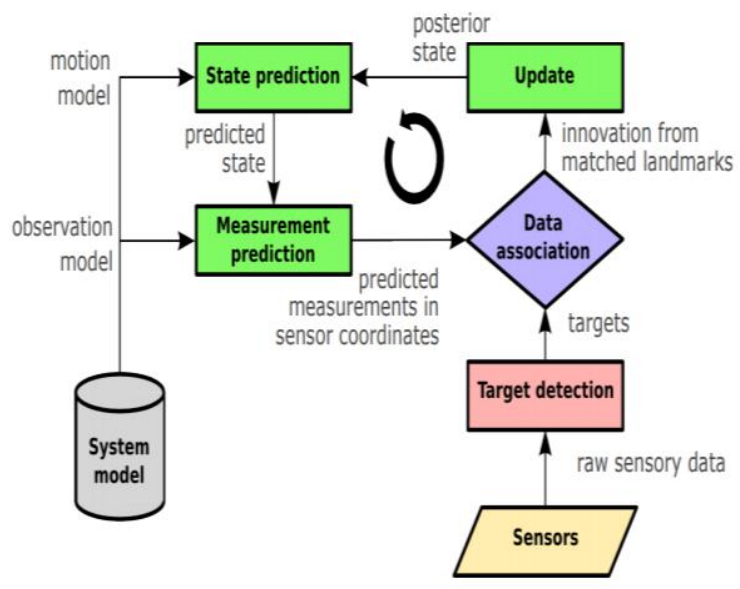

Fig 1: Kalman filter cycle

\section{TOOL ALGORITHMS FOR ESTIMATION}

If we get an idea of where an object is in the image because we have an idea of the motion from previous images, we need less work detecting or recognizing the object.

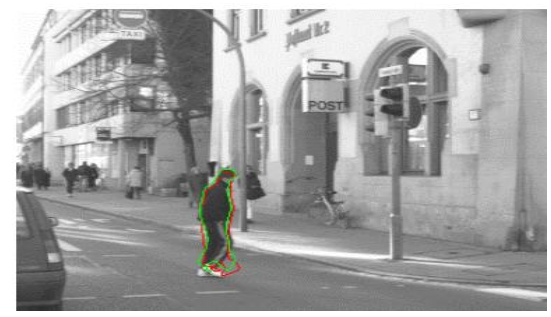

Fig 2: Tracking Process

The crowd density estimation is an important criterion for the validation of our proposed tools. This part of the paper considers the role of automatic estimations of crowd density after import the video of any extension and their significance for the automatic monitoring of motion able areas where crowds or moveable parts are expected to be present. A new technique is proposed which is able to estimate crowd ranging from very low to very high concentrations of people. This technique is based on the kalman filter (kalman designs a Kalman filter or Kalman state estimator given a state-space model of the plant and the process and measurement noise covariance data. The Kalman estimator provides the optimal solution to the following continuous or discrete estimation problems) and differences of texture muster on the images of crowds. Images of low density crowds exhibits rough textures, while images with high densities tend to present finer textures.

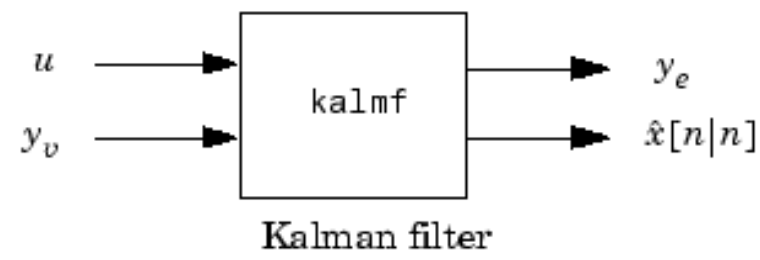

The inputs of kalmf are $u$ and $y_{v}$. the output are ye and $x[n][n]$. To attach the kalman filter for crwod estimation we use the following function

kalmanFilter=configureKalmanFilter('ConstantAcceleration',[1 00 120],[1 111$] * 1 \mathrm{e} 5,[25,10,10], 25)$;

\subsection{Video Input}

A video is basically a collection of continuous images displayed at a certain rate (generally 30 frames per second). To extract the frames from video first we need to attach this video to the input stream and then extract those as and when required. To attach the video to input stream we use the following function

\%obj.reader = vision.VideoFileReader('b.avi'); \%obj.reader = vision.VideoFileReader('c.avi'); \%obj.reader $=$ vision. VideoFileReader('d.avi');

Viedo b.avi, c.avi and d.avi are used as a vieod database and tool are developed in MATLAB and result record and shown the snapshot in below figureand also shown the algorthim step for crowd estimation with kalman filter with two model. 

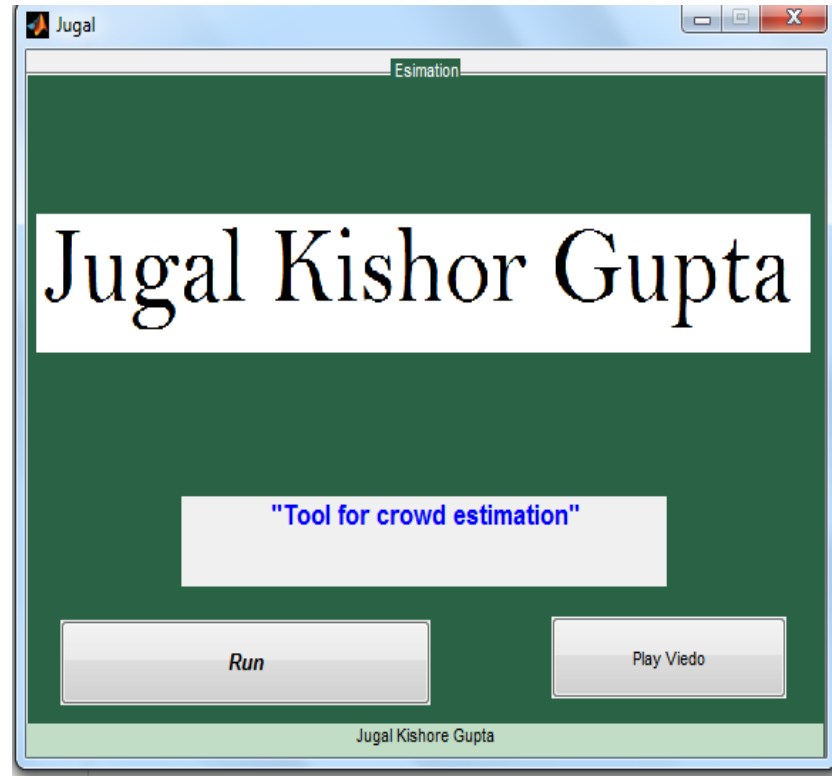

Fig 3: Estimation Tool

Step 1: Create or Read a video or moving object \%obj.reader = vision.VideoFileReader('d.avi'); Step 2: Initialize Tracks or state of moving object Step 3: Detect moving objects from the video, and track them across video frames

Step 4: Create a Kalman filter object

Step 5: Display Count Resuls in Video Frame

\section{RESULT}

MotionModel

ConstantVelocity:- Constant target velocity assumption : Useful to model smooth target motion.

Table 1: Result with ConstantVelocity

\begin{tabular}{|l|l|l|}
\hline Video Name & Exact Object & Estimated Object \\
\hline a.avi & 4 & 3 \\
\hline b.avi & 10 & 7 \\
\hline c.avi & 7 & 7 \\
\hline d.avi & 10 & 17 \\
\hline
\end{tabular}

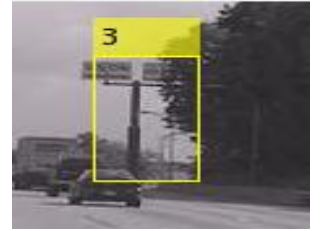

Fig 4: a.avi

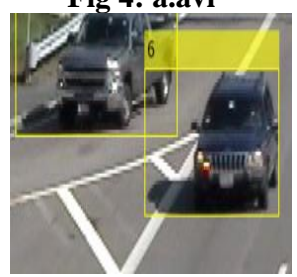

Fig 6: c.avi

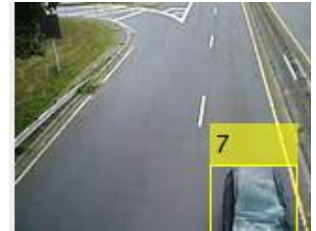

Fig 5: b.avi

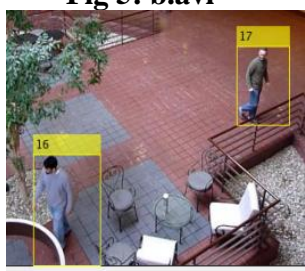

Fig 7: d.avi
ConstantAcceleration:- Constant target acceleration assumed: Useful to model target motion that is smooth in position and velocity changes
Table 2: Result with ConstantAcceleration

\begin{tabular}{|l|l|l|}
\hline Video Name & Exact Object & Estimated Object \\
\hline a.avi & 4 & 3 \\
\hline b.avi & 10 & 7 \\
\hline c.avi & 7 & 7 \\
\hline d.avi & 10 & 15 \\
\hline
\end{tabular}

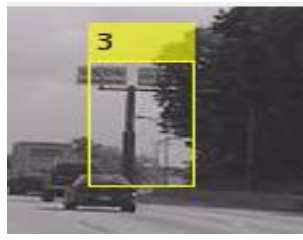

Fig 8 a.avi

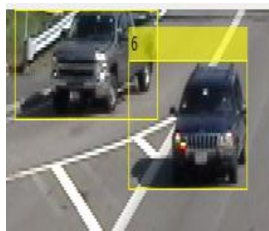

Fig 10: c.avi

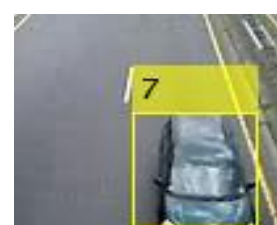

Fig 9:b.avi

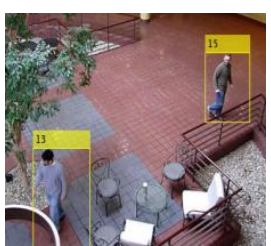

Fig 11: d.avi

\section{CONCLUSION AND FUTURE SCOPE}

In this paper two motion models have been discused and implemented in MATLAB, in result section both motion model (ConstantVelocity and ConstantAcceleration) results are shown in tabular and results are good and more accurate for ConstantAcceleration motion model. In future proposed a new index parameter for measurement of accuracy for crowd density estimation.

\section{REFERENCES}

[1] Beril Sirmacek, Peter Reinartz, Kalman Filter Based Feature Analysis For Tracking People From Airborne Images, German Aerospace Center (DLR), Remote Sensing Technology Institute PO Box 1116, 82230, Wessling, Germany

[2] Ming Jiang, Jingcheng Huang, Xingqi Wang, Jingfan Tang, Chunming Wu, An Approach for Crowd Density and Crowd Size Estimation JOURNAL OF SOFTWARE, VOL. 9, NO. 3, MARCH 2014.

[3] A.N. Marana, S.A. Velastin, L.F. Costa, R.A.Lotufo, Automatic Estimation of Crowd Density Using Texture", International Workshop on Systems and Image Processing, IWSIP'97, May 28-30, Poland.

[4] Mohamed H. Dridi, "Tracking Individual Targets in High Density Crowd Scenes Analysis of a Video Recording in Hajj 2009",Current Urban Studies, 2015, 3, 35-53 Published Online March 2015 in SciRes.

[5] Shilin Zhang and Xunyuan Zhang "Crowded Pedestrian Detection and Density Estimation by Visual Words Analysis", International Journal of Multimedia and Ubiquitous Engineering Vol. 10, No. 3 (2015), pp. 99 $108 \mathrm{http} / / / \mathrm{dx}$. doi.org/10.14257/ijmue.2015.10.3.10.

[6] T.S.Surendiran , G.Michael, S.P.Vijayaragavan "Crowd Density Estimation Using Accumulated Mosaic Image Difference" International Journal Of Engineering Sciences \& Research Technologyissn: 2277-9655 Scientific Journal Impact Factor: 3.449 (Isra), Impact Factor: 2.114. 
[7] Kai Arras, Cyrill Stachniss, Maren Bennewitz, Wolfram Burgard, Robotics 2 Target Tracking, Gian Diego Tipaldi, v.1.1, Jan 2012.

[8] Schofer J. Ushpiz A. Polus, A. Pedestrian Flow and Level of Service. J. Transportation Eng, 109(1):46-56, 1983.

[9] Zi Ye, Jinqiao Wang, Zhenchong Wang, Hanqing Lu. Multiple features fusion for crowd density estimation. Proceeding ICIMCS '12 Proceedings of the $4^{\text {th }}$ International Conference on Internet Multimedia Computing and Service, pp. 42-45, 2012.

[10] Subburaman V B, Descamps A, Carincotte C. Counting People in the Crowd Using a Generic Head Detector[C].
Proceedings of 2012 IEEE 9th International Conference on Advanced Video and Signal-Based Surveillance (AVSS): September 18-21, 2012. Beijing, China, pp. 470-475, 2012

[11] J.F. Dickie, "Major crowd catastrophes", Safety Science, vol. 18, pp. 309-320, 1995

[12] Kumar, T. and K. Verma, 2010a. A theory based on conversion of RGB image to gray image. Int. J. Computer. Appli., 7: 5-12. DOI: 10.5120/1140-1493.

[13] J.D. Sime, "Crowd psychology and engineering", Safety Science, vol. 21, pp. 1-14, 1995.

[14] R.C. Gonzales, R. Woods, Digital Image Processing, Addison-Wesley Publishing Company, 1993. 